\title{
Reduced migration of Ishikawa cells associated with downregulation of aquaporin-5
}

\author{
XIU XIU JIANG ${ }^{1}$, KAI HONG XU ${ }^{1}$, JUN YAN MA ${ }^{2}$, YONG HONG TIAN ${ }^{1}$, \\ XIAO YAN GUO ${ }^{2}$, JUN LIN ${ }^{1}$ and RUI JIN WU ${ }^{1}$ \\ Departments of ${ }^{1}$ Gynecology and Obstetrics and ${ }^{2}$ Laboratory of Gynecology and Obstetrics, Women's Hospital, \\ School of Medicine, Zhejiang University, Hangzhou, Zhejiang 310006, P.R. China
}

Received January 17, 2012; Accepted May 14, 2012

DOI: $10.3892 / \mathrm{ol} .2012 .738$

\begin{abstract}
Aquaporin (AQP)-dependent cell migration has broad implications in angiogenesis, tumor metastasis, wound healing, glial scarring and other events requiring cell movement. There are 13 isoforms of AQP (0-12) that have been identified in mammals. It is unclear whether AQP5 plays a role in the development of endometrial cancer. We recently demonstrated that ovarian steroids may affect the expression of AQP5 in the female genital tract. In this study, we considered whether AQP5 may affect cell migration in Ishikawa cells, an adenocarcinoma cell line derived from the endometrium. The results showed that the downregulation of AQP5 results in reduced Ishikawa cell migration. The estrogen (E2) receptor in the promoter of AQP5 mediated the regulation of AQP5 expression in the normal endometrium and endometrial cancer. By contrast, the upregulation of AQP5 by E2 increased cell migration, invasion and adhesion through increased annexin-2, which is responsible for F-actin remodeling and rearrangement. E2 regulates Ishikawa cell migration by regulating the AQP5 expression.
\end{abstract}

\section{Introduction}

Aquaporins (AQPs) are membrane glycoproteins, embedded in cell membranes, that allow water to move in response to osmotic and hydrostatic differences (1). There are at least 13 AQPs (0-12) in mammals expressed in a number of epithelia, endothelia and other types of cells. At least 12 different tumor cell types have been reported to express AQPs in vivo in humans and rodents. Accumulating evidence suggests that

Correspondence to: Dr Rui Jin Wu, Department of Gynecology and Obstetrics, Women's Hospital, School of Medicine, Zhejiang University, 2 Xue Shi Road, Hangzhou, Zhejiang 310006, P.R. China

E-mail: janet418@hotmail.co.uk

Key words: aquaporin-5, Ishikawa cells, cell migration, small interfering RNA, transwell chamber ovarian steroids may affect the expression of several AQPs in the female genital tract (2-3). AQP-dependent cell migration has broad implications in angiogenesis, tumor metastasis, wound healing, glial scarring, embryonic development and other events requiring cell movement (4).

AQP5 is expressed in the mouse, rat, pig and human uterus (5-7). AQP5 has been shown to be involved in cell proliferation and carcinoma genesis in lung and colon tissues $(8,9)$. Results of previous studies demonstrated that in ovarian cancer, AQP5 is important in a variety of pathological conditions, including ascites formation (10). In addition, AQP5 is expressed in normal human endometrium (11) and endometriosis ectopic endometrium (7). The expression of human endometrial AQP5 is menstrual cycle-dependent (7). High levels of AQP5 are found at the proliferative and mid-secretory phases and are positively correlated with serum levels of estradiol (E), suggesting that estrogen (E2) may regulate the expression of AQP5 in endometrial cells (7).

Migration is crucial in endometriosis and endometrial cancer development. However, it is unclear whether the downregulation of AQP5 is directly correlated with endometrial disease. In this study, we examined the role of AQP5 in cell migration in human Ishikawa cells, an endometrial adenocarcinoma cell line (12).

Endometrial carcinoma (EC) is the most common type of gynecological cancer in developed countries. EC is divided into two subtypes: type I, endometrioid-type EC, which accounts for $80-90 \%$ of EC, is of endometrial origin and is estrogen-dependent, and type II, non-endometrioid type $\mathrm{EC}$, is mostly presented by papillary serous and clear-cell adenocarcinomas, accounts for $10-20 \%$ of EC and is usually estrogen-independent (13). Although it is a common malignancy, the molecular aspects of EC are poorly understood. For certain tumors, positive correlations have been established between histological tumor grade and the amount of AQP expression $(14,15)$. AQP-dependent cell migration has broad implications in angiogenesis, tumor metastasis, wound healing, glial scarring and other events requiring rapid cell movement (16-18). It remains unclear whether AQP5 mediates these processes. Direct mechanistic evidence for AQP regulation of EC cell migration and invasion in the context of EC is limited. Therefore, we aimed to determine whether AQP5 is involved in EC development by migration. 


\section{Materials and methods}

Cells culture. Ishikawa cells (American Type Culture Collection, Manassas, VA, USA), an endometrial adenocarcinoma cell line, provided by the Laboratory of Gynecology and Obstetrics, Women's Hospital, School of Medicine, Zhejiang University, China, were cultured in phenol red RPMI-1640 medium (Thermo Scientific HyClone, South Logan, UT, USA), supplemented with $10 \%$ fetal bovine serum (FBS; $\mathrm{vol} / \mathrm{vol}), 100 \mathrm{U} / \mathrm{ml}$ penicillin and $100 \mathrm{U} / \mathrm{ml}$ streptomycin at $37^{\circ} \mathrm{C}$ plus $5 \% \mathrm{CO}_{2}$.

RNA interference (RNAi) experiments. Cells were seeded at $1 \times 10^{-6} /$ well in 6 -well plates. When the cells reached $80-90 \%$ confluence, cationic lipid complexes, prepared by incubating RNAi or negative RNAi with $5 \mathrm{nM}$ Lipofectamine 2000 (Invitrogen Life Technologies, Carlsbad, CA, USA) in $500 \mu \mathrm{l}$ RPMI-1640 medium, were added into the wells according to the manufacturer's instructions. Cells were suspended and cultured in RPMI-1640. The study groups were experimental [group 1: specific small interfering RNA (siRNA)], control (group 2: non-specific siRNA) and blank (group 3: no siRNA). After $48 \mathrm{~h}$, green fluorescence was quantified by a fluorescence-activated cell sorting (FACS) analysis to assess the transfection efficiency. The efficiency of transfection was confirmed by real-time PCR (RT-PCR) and western blotting. AQP5 siRNA duplexes were chemically synthesized by Thermo Scientific Dharmacon (Lafeyette, CO, USA). Four siRNAs targeting human AQP5 and four non-specific siRNAs each combined into one pool were designed and synthesized by Dharmacon: Duplex 1, 3'-GCUCCGGGCUUUCUUCUA CUU-5'; duplex 2, 3'-GAACCCAGCCCGCUCUUUUUU-5'; duplex 3, 3'-CGUAUGAGCCUGACGAGGAUU-5'; duplex 4, 3'-GCGCUCAACAACAACACAAUU-5'. Non-specific control duplexes: Duplex 1, 3'-AUGAACGUGAAUUGCU CAA-5'; duplex 2, 3'-UAAGGCUAUGA AGAGAUAC-5'; duplex 3, 3'-AUGUAUUGGCCUGUAUUAG-5'; duplex 4, 3'-UAGCGACUAAACACAUCAA-5'.

Quantitative RT-PCR. Two days after transfection, total RNA was extracted at the indicated times using an EZ spin column RNA extraction kit (Sangon, Shanghai, China). cDNA was reverse transcribed using an MMLV first-strand synthesis kit (BBInternational, Madison, WI, USA). PCR reactions were conducted in a $25 \mu 1$ volume, containing $11 \mu \mathrm{lDNA}, 1 \mathrm{mM}$ of each forward and reverse primer and $0.25 \mathrm{xSyBr}$ green mix. $\beta$-actin was used as the internal control to quantitate initial cell transcripts. Primer sequences included: $\beta$-actin; sense, 5'-CCTGTACGCCAACACAGTG-C-3'; antisense, 5'-ATAC TCCTGCTTGCTGATCC-3'; AQP5; sense, 5'-CTGTCCATT GGCCTGTCTGTC-3'; antisense, 5'-GGCTCATACGTGCC TTTGATG-3'. Quantitative real-time PCR analysis was conducted using an Applied Biosystems 7500 fast RT-PCR system (ABI; Carlsbad, CA, USA).

Western blot analysis. Cells were harvested and re-suspended in PBS 2 days after transfection. Total protein was extracted using a RIPA kit. GADPH (EarthOx, San Fransisco, CA, USA) was used as reference for the normalized expression level. The protein was electrophoresed on a polyacrylamide gel and transferred to a Hybond-C nitrocellulose membrane. Briefly, the separated samples were transferred to nitrocellulose membranes and exposed to mouse anti-AQP5 antibody (1:1000) for $2 \mathrm{~h}$, followed by horseradish peroxidase-conjugated goat anti-mouse IgG antibody $(1: 10,000)$ for $1.5 \mathrm{~h}$ at room temperature. Proteins were visualized using enhanced chemiluminescence (ECL) detection reagents (Santa Cruz Biotechnology, Santa Cruz, CA, USA). The antibodies were obtained from Abcam.

Transwell assay. Ishikawa cells $\left(1 \times 10^{5} /\right.$ well $)$ were loaded and cultured in 24-well plates. Cells were vaccinated in the transwell upper chamber in a small room at $37^{\circ} \mathrm{C}$ with a $5 \%$ $\mathrm{CO}_{2}$ incubator; the upper chamber contained $1 \%$ serum and the lower chamber contained $10 \%$ serum. Incubated cells were removed from the small room at $24,48,72$ and $96 \mathrm{~h}$. The lower cell medium was removed and crystal violet was stained before the cells were counted. Each count of five high-power field was averaged and the number of cell perforations formed in each group was calculated.

Statistical analysis. Data were normally distributed and were shown as the mean \pm SEM. The independent-samples t-test was used to evaluate the statistical significance of the differences between two groups, and the one-way ANOVA test was used to evaluate the statistical significance of the differences between more than two groups. SPSS version 16.0 (SPSS Inc., Chicago, IL, USA) was used for the statistical analysis. $\mathrm{P}<0.05$ was considered to indicate a statistically significant difference.

\section{Results}

AQP5 gene expression in Ishikawa cells. After $48 \mathrm{~h}$, cultured cells were attached onto the thin film. RT-PCR amplification of the AQP5 gene in the Ishikawa cells was examined by $1 \%$ agar gel electrophoresis, which showed the expression of AQP5 mRNA in Ishikawa cells. The AQP5 molecular length was approximately $250 \mathrm{bp}$. We detected AQP5 protein levels in Ishikawa cells using western blotting. The results demonstrated a higher amount of AQP5 protein expression in Ishikawa cells.

Effect of RNAi on AQP5 gene expression levels in Ishikawa cells. RT-PCR demonstrated the level of interference effects in AQP5 mRNA. Compared with the control and blank groups, mRNA expression levels in the experimental group were significantly decreased $(\mathrm{P}=0.03)$ (Table I). In the Ishikawa cells, after AQP-5 RNAi, the protein expression level was significantly decreased (group 1 vs. groups 2 and $3, \mathrm{P}=0.007$ ), while AQP5 protein expression in the control and blank group was not significantly different (group 2 vs. 3 , $\mathrm{P}=0.507$ ) (Table II).

Determination of Ishikawa cell migration after RNAi. Following a comparison of the experimental and negative group, we determined that an optimal transfection efficiency of $80 \%$ could be achieved at $48 \mathrm{~h}$; the best concentration was $100 \mathrm{nM}$. When cells were transfected with $100 \mathrm{ng}$ siRNA in the transwell chamber after $24,48,72$ and $96 \mathrm{~h}$, the number of perforated cells increased with time. Following a comparison 
Table I. AQP5 mRNA expression in Ishikawa cells after RNA interference.

\begin{tabular}{lccc}
\hline Group & Sample (mean \pm SD) & $\beta$-actin (mean \pm SD) & P-value \\
\hline Experimental (group 1) & $21.638 \pm 0.078$ & $21.778 \pm 0.028$ & $0.03^{\mathrm{a}}$ \\
Control (group 2) & $27.324 \pm 0.037$ & $21.155 \pm 0.069$ & $0.48^{\mathrm{b}}$ \\
Blank (group 3) & $29.136 \pm 0.046$ & $20.672 \pm 0.083$ &
\end{tabular}

${ }^{\mathrm{a} C}$ Comparison of group 1 vs. groups 2 and 3. ${ }^{\mathrm{b} C o m p a r i s o n}$ of group 2 vs. group 3. AQP, aquaporin; SD, standard deviation.

Table II. AQP5 protein levels in Ishikawa cells after RNA interference examined by western blotting.

\begin{tabular}{lccc}
\hline Group & Expression amount (mean \pm SD) & Inhibition rate (AQP5/GADPH) $(\%)$ & P-value \\
\hline Experimental (group 1) & $0.222 \pm 0.108$ & 75.84 & $0.007^{\mathrm{a}}$ \\
Control (group 2) & $0.844 \pm 0.166$ & 8.16 & 0.507 \\
Blank (group 3) & $0.919 \pm 0.129$ & & \\
\hline
\end{tabular}

${ }^{\mathrm{a} C o m p a r i s o n ~ o f ~ g r o u p ~} 1$ vs. groups 2 and 3 . ${ }^{\mathrm{b}}$ Comparison of group 2 vs. 3 . AQP, aquaporin; SD, standard deviation.

Table III. Cell perforation ability after RNA interference.

\begin{tabular}{|c|c|c|c|c|c|c|c|c|}
\hline \multirow[b]{2}{*}{ Group } & \multicolumn{4}{|c|}{ Number of cell perforations } & \multirow[b]{2}{*}{ P-value ${ }^{a}$} & \multirow[b]{2}{*}{ P-value ${ }^{b}$} & \multirow[b]{2}{*}{ P-value } & \multirow[b]{2}{*}{ P-value } \\
\hline & $24 \mathrm{~h}$ & $48 \mathrm{~h}$ & $72 \mathrm{~h}$ & $96 \mathrm{~h}$ & & & & \\
\hline 1 & $35.7 \pm 4.8$ & $54.6 \pm 1.1$ & $70.4 \pm 2.3$ & $76.7 \pm 4.4$ & 0.37 & 0.04 & 0.03 & 0.01 \\
\hline 2 & $34.33 \pm 4.3$ & $96.6 \pm 3.1$ & $140.5 \pm 2.3$ & $160.9 \pm 5.2$ & 0.74 & 0.83 & 0.66 & 0.58 \\
\hline 3 & $32.3 \pm 4.5$ & $98.5 \pm 2.3$ & $150.2 \pm 7.1$ & $179.6 \pm 6.3$ & & & & \\
\hline
\end{tabular}

Group 1 vs. groups 2 and 3 at ${ }^{\mathrm{a}} 24 \mathrm{~h},{ }^{\mathrm{b}} 48 \mathrm{~h},{ }^{\mathrm{c}} 72 \mathrm{~h}$ and ${ }^{\mathrm{d}} 96 \mathrm{~h}$.

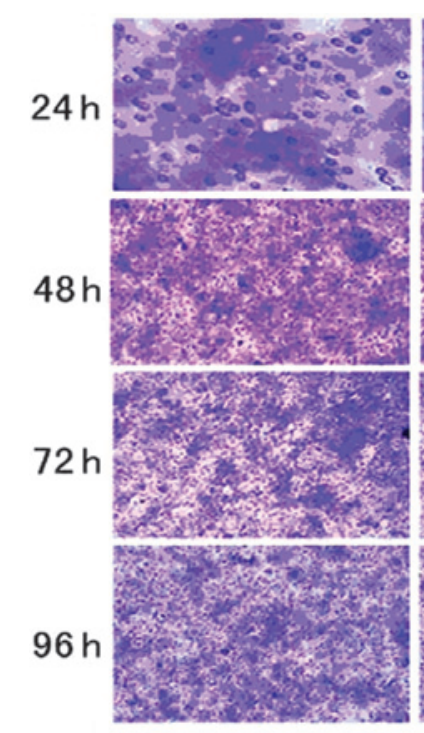

Blank

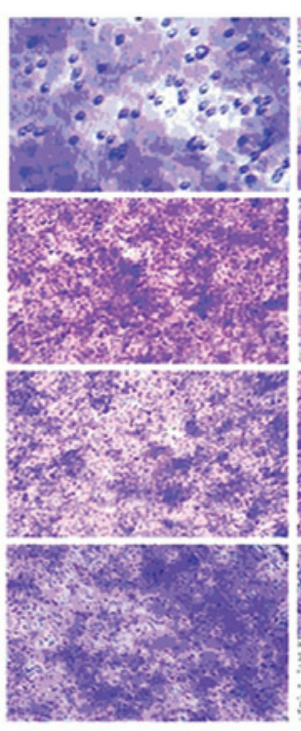

Control

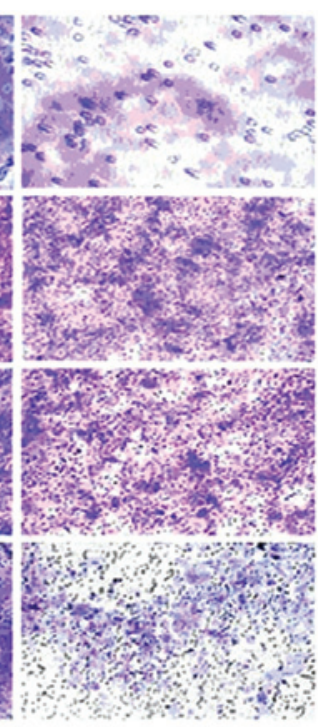

Experimental

Figure 1. Increased migration of AQP5-expressing Ishikawa cells. Control and AQP5-knockdown tumor cells were applied to transwell filters, were stained in crystal violet in lower room cells on different days and counted in low/high-power fields. At $24 \mathrm{~h}$; original magnification, $\mathrm{x} 400$; at $48-96 \mathrm{~h}$; original magnification, x200.Calculated as the average number of cells. AQP, aquaporin. 
of the experimental group with control and blank groups at 48,72 and $96 \mathrm{~h}$, the number of perforated cells in the experimental group decreased (Table III, Fig. 1).

\section{Discussion}

Accumulating evidence has demonstrated that AQPs are important for cell migration, invasion and spread in malignancy (19). Expression of AQP1 has been associated with colon cancer, mammary carcinoma, brain tumor, hemangioblastoma and multiple myeloma (20). Other studies have demonstrated an increased expression of AQP3 in skin carcinoma, increased AQP4 expression in glioblastoma and increased AQP5 expression in pancreatic and colon cancer (21-24).

Cell migration is affected by numerous factors; it is time-dependent (25), significantly enhanced by collagen IV in embryonic stem cells (26), and it has been observed that even a lesion can induce an increase in the migration of human neural progenitor cells (27). The findings from this study demonstrate that AQP5 knockdown reduced the migration of EC cells, suggesting that AQP5 is involved in the development of EC. The mechanisms underlying AQP-facilitated migration, invasion and proliferation remain unclear. Certain data suggest that changes in the cell volume and shape mediated by AQP may contribute to migration and invasion (28). The present study demonstrated that inhibition of the endogenous AQP5 expression attenuated migration of EC cells as examined by a transwell assay, providing evidence that supports our hypothesis (3).

In a previous study, we found that AQP5 was highly expressed in endometriosis patients in the eutopic and ectopic endometria, and expression levels changed with the E2 level. The present study provides evidence that AQP5 is expressed in endometroisis and endometrial cancer cells, both of which are E2-dependent diseases (29).

Several studies have revealed an estrogen response element (ERE) in the promoter of AQP2, which mediates the regulation of $\mathrm{AQP} 2$ expression by $\mathrm{E}$ in the normal endometrium and EC. By contrast, the upregulation of AQP2 by E2 increased cell migration, invasion and adhesion through increased annexin-2, which is responsible for F-actin remodeling and rearrangement (3). We consider that these results may provide insights into the potential mechanism in which AQP5 regulates migration by E2.

AQP-facilitated cell migration to different cell types suggests that AQP expression in tumor cells may increase local tumor invasiveness and the ability of tumor cells to metastasize by crossing plasma membrane barriers. To test this possibility, tumor cell migration, invasiveness and metastatic potential were evaluated by comparing Ishikawa cell lines with and without AQP5 expression. In vitro analysis of cell migration using transwell migration assays demonstrated a greatly increased migration of AQP5-expressing tumor cells, as predicted. From counting numerous cells on multiple filters, the ratio of AQP5-expressing cells compared with control cells was significantly increased after $48 \mathrm{~h}$, indicating increased migration of AQP5-expressing cells. The involvement of AQP5 in tumor cell migration has potentially important clinical implications. It provides a functional explanation for $\mathrm{AQP}$ expression in tumor cells and for the correlations between tumor shift and AQP expression. Additionally, it provides a rational basis to evaluate $\mathrm{AQP}$ inhibitors, when available, for tumor therapy, both for reduction of tumor development and tumor spread (30).

\section{References}

1. Verkman AS: More than just water channels: unexpected cellular roles of aquaporins. J Cell Sci 118: 3225-3232, 2005.

2. Aralla M, Borromeo V and Groppetti D: A collaboration of aquaporins handles water transport in relation to the estrous cycle in the bith uterus. Theriogenology 72: 310-321, 2009

3. Zou LB,Zhang RJ, Tan YJ, et al: Identification of estrogen response element in the aquaporin-2 gene that mediates estrogen-induced cell migration and invasion in human endometrial carcinoma. J Clin Endocrinol Metab 96: 1399-1408, 2011

4. Chang HL, Pieretti-Vanmarcke R, Nicolaou F, et al: Mullerian inhibiting substance inhibits invasion and migration of epithelial cancer cell lines. Gynecol Oncol 120: 128-134, 2011.

5. Asari T, Maruyama K and Kusama H: Salivation triggered by pilocarpine involves aquaporin-5 in normal rats but not in irradiated rats. Clin Exp Pharmacol Physiol 36: 531-538, 2009.

6. Skowronski MT, Kwon TH and Nielsen S: Immunolocalization of aquaporin 1,5 and 9 in the female pig reproductive system. J Histochem Cytochem 57: 61-67, 2009.

7. Jiang XX, Wu RJ, Xu KH, Zhou CY, Guo XY, Sun YL and Lin J: Immunohistochemical detection of aquaporin expression in eutopic and ectopic endometria from women with endometriomas. Fertil Steril 94: 1229-1234, 2010.

8. Machida Y, Ueda Y, Shimasaki M, Sato K, Sagawa M, Katsuda S and Sakuma T: Relationship of aquaporin 1, 3, and 5 expression in lung cancer cells to cellular differentiation, invasive growth, and metastasis potential. Hum Pathol 42: 669-678, 2011.

9. Kang SK, Chae YK, Woo J, et al: Role of human aquaporin 5 in colorectal carcinogenesis. Am J Pathol 173: 518-525, 2008.

10. Yang JH, Shi YF, Cheng Q and Deng L: Expression and localization of aquaporin-5 in the epithelial ovarian tumors. Gynecol Oncol 100: 294-299, 2006.

11. He RH, Sheng JZ, Luo Q, et al: Aquaporin-2 expression in human edometrium correlates with serum ovarian steroid hormones. Life Sci 79: 423-429, 2006.

12. Schaefer LWR, Fischer WR, et al: In vitro-Ishikawa cell test for assessing tissue-specific chemical effects on human endometrium. Reprod Toxicol 30: 89-93, 2010.

13. Ryan AJ, Susil B, Jobling TW and Oehler MK: Endometrial cancer. Cell Tissue Res 322: 53-61, 2005.

14. Warth A, Kröger S and Wolburg H: Redistribution of aquaporin-4 in human glioblastoma correlates with loss of agrin immunoreactivity from brain capillary basal laminae. Acta Neuropathol 107: 311-318, 2004.

15. Saadoun S, Papadopoulos MC, Davies DC, Krishna S and Bell BA: Aquaporin-4 expression is increased in oedematous human brain tumours. J Neurol Neurosur Psychiatry 72: 262-265, 2002.

16. Ruiz-Ederra J and Verkman AS: Aquaporin-1 facilitated keratocyte migration in cell culture and in vivo corneal wound healing models. Exp Eye Res 89: 159-165, 2009.

17. Ding T, Gu F, Fu L and Ma YJ: Aquaporin-4 in glioma invasion and analysis of molecular mechanisms. J Clin Neurosci 17: 1359-1361, 2010.

18. Hayashi S, Takahashi N, Kurata N, Yamaguchi A, Matsui H, Kato $\mathrm{S}$ and Takeuchi K: Involvement of aquaporin-1 in gastric epithelial cell migration during wound repair. Biochem Bioph Res Commun 386: 483-487, 2009.

19. Papadopoulos MC, Saadoun S and Verkman AS: Aquaporins and cell migration. Pflug Arch 456: 693-700, 2008.

20. Nico B and Ribatti D: Aquaporins in tumor growth and angiogenesis. Cancer Lett 294: 135-138, 2010.

21. Hara-Chikuma M and Verkman AS: Prevention of skin tumorigenesis and impairment of epidermal cell proliferation by targeted aquaporin-3 gene disruption. Mol Cell Biol 28: 326-332, 2008.

22. McCoy E and Sontheimer H: Expression and function of water channels (aquaporins) in migrating malignant astrocytes. Glia 55: 1034-1043, 2007.

23. Hara-Chikuma $M$ and Verkman AS: Aquaporin-3 facilitates epidermal cell migration and proliferation during wound healing. J Mol Med (Berl) 86: 221-231, 2008

24. Ding T, Ma Y, Li W, Liu X, Ying G, Fu L and Gu F: Role of aquaporin-4 in the regulation of migration and invasion of human glioma cells. Int J Oncol 38: 1521-1531, 2011. 
25. Assis ACM, Carvalho JL, Jacoby BA, et al: Time-dependent migration of systemically delivered bone marrow mesenchymal stem cells to the infarcted heart. Cell Transplant 19: 219-230, 2010.

26. Li HY, Liao CY, Lee KH, et al: Collagen IV significantly enhances migration and transplantation of embryonic stem cells: involvement of alpha 2 beta 1 integrin-mediated actin remodeling. Cell Transplant 20: 893-907, 2011.

27. Behrstock S, Ebert AD, Klein S, Schmitt M, Moore JM and Svendsen CN: Lesion-induced increase in survival and migration of human neural progenitor cells releasing GDNF. Cell Transplant 17: 753-762, 2008.
28. Huebert RC, Vasdev MM, Shergill U, et al: Aquaporin-1 facilitates angiogenic invasion in the pathological neovasculature that accompanies cirrhosis. Hepatology 52: 238-248, 2010.

29. Kirchebner P, Marth C, Mayer I and Daxenbichler G: Metabolism of E1 and E2 in Ishikawa endometrium carcinoma cells: influence of TNFa. J Steroid Biochem Mol Biol 39: 221-222, 1991.

30. López-Campos JL, Sánchez Silva R, Gómez Izquierdo L, et al: Overexpression of aquaporin-1 in lung adenocarcinomas and pleural mesotheliomas. Histol Histopathol 26: 451-459, 2011. 